\title{
Tipología sociométrica de niños y niñas de Chile en educación primaria. Un análisis por sexo y curso académico
}

\author{
Sociometric Typology of Chilean Children of Primary Education. An Analysis by Sex and \\ Academic Course
}

\section{Tipologia sociométrica das crianças chilenas do ensino fundamental. Uma análise por sexo e curso acadêmico}

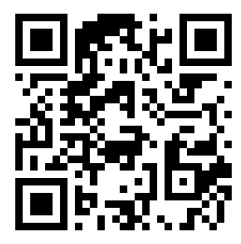

Nelly Gromiria Lagos-San Martín

Universidad del Bío-Bío

Chillán, Chile

nlagos@ubiobio.cl

https://orcid.org/0000-0002-2029-5219

Ximena Soto-Soto

Universidad del Bío-Bío

Chillán, Chile

xime25530@gmail.com

https://orcid.org/0000-0002-8641-0961

Recibido • Received • Recebido: 17 / 08 / 2018

Corregido • Revised • Revisado: 12 / 08 / 2019

Aceptado • Accepted • Aprovado: 07/ 11 / 2019

\begin{abstract}
Resumen: La competencia social como capacidad para crear y mantener relaciones saludables supone comportamientos valorados positivamente por la comunidad en la que un sujeto se desenvuelve. En la escuela, la competencia social cobra una gran importancia, por lo que se estima necesario analizar las tipologías sociométricas del estudiantado en función del sexo y curso académico. En esta investigación participaron 772 estudiantes de 26 cursos diferentes, pertenecen a 8 comunas de la región de Ñuble, 420 niños (54.4 \%) y 352 niñas (45.6), 250 cursan $4^{\circ}$ año, 274 cursan $5^{\circ}$ año y 248 cursan $6^{\circ}$ año de primaria. El instrumento utilizado es el Cuestionario sociométrico de nominaciones entre iguales, instrumento que proporciona información sobre la niñez rechazada, ignorada, controvertida y medios preferidos en sus cursos. Los datos fueron analizados con el software SOCIOMET. Los resultados indican un mayor porcentaje de niños rechazados, ignorados y preferidos, que de niñas en estas tipologías, hecho que se reitera también por curso académico. Se puede observar, además, que los índices de amistad en los distintos cursos superan los índices de enemistad. A partir de estos resultados, se estima que pueden proyectarse intervenciones ajustadas a los requerimientos contextuales.
\end{abstract}

Palabras claves: Competencia social; educación básica; SOCIOMET. 
doi: http://doi.org/10.15359/ree.24-1.8

URL: http://www.una.ac.cr/educare

CORREO: educare@una.cr

\begin{abstract}
Social competence, seen as a capacity to create and maintain healthy relationships, involves behaviors valued positively by the community in which a subject develops. At school, the social competence acquires great importance; this is the reason why analyzing the sociometric typologies of the students according to the sex and academic course is considered necessary. This research involved 772 students from 26 different classrooms; they live in eight communities in the Ñuble region. There were 420 children (54.4\%) and 352 girls (45.6\%); of them, 250 children are in 4th grade, 274 in 5th grade, and 248 in 6th grade of elementary school. The instrument used is the Sociometric Questionnaire of peer nominations; it provides information about children who are rejected, ignored, and controversial; it also collects information about the average of preferred children in classrooms. The data were analyzed with the SOCIOMET software. The results indicate a higher percentage of rejected, ignored, and preferred boys than of girls in these typologies; this fact is also reiterated by the academic year. Besides, the indices of friendship in the different classrooms surpass the indices of enmity. Based on these results, interventions tailored to contextual requirements can be projected.
\end{abstract}

Keywords: Social competence; basic education; SOCIOMET.

Resumo: A competência social como capacidade de criar e manter relacionamentos saudáveis envolve comportamentos valorizados positivamente pela comunidade em que um sujeito se desenvolve. Na escola, a competência social adquire uma grande importância pelo que se considera necessário analisar as tipologias sociométricas dos estudantes segundo o sexo e o curso acadêmico. Esta pesquisa envolveu 772 estudantes de 26 cursos diferentes, que pertencem a 8 municípios na região de Dims, 420 meninos (54.4\%) e 352 meninas (45.6\%), deles 250 crianças matriculadas no $4^{\circ}$ ano, 274 no $5^{\circ}$ ano e 248 estão no $6^{\circ}$ ano do ensino fundamental. O instrumento utilizado é o Questionário Sociométrico de indicações de pares; instrumento que fornece informações sobre crianças rejeitadas, ignoradas, controversas, e os meios preferidos nos seus cursos. Os dados foram analisados com o software SOCIOMET. Os resultados indicam uma porcentagem maior de meninos rejeitados, ignoradas e preferidas que as meninas, dentro dessas tipologias, fato que também é reiterado no ano letivo. Observa-se, ademais, que os índices de amizade nos diferentes cursos superam os índices de inimizade. Com base nesses resultados, estima-se que as intervenções adaptadas aos requisitos contextuais possam ser projetadas.

Palavras-chave: Competência social; educação básica; SOCIOMET.

\title{
Antecedentes
}

La competencia social, entendida como capacidad para crear y mantener buenas relaciones, favorece el bienestar personal de un sujeto en su medio social. Esta competencia exige comportamientos valorados positivamente por la comunidad en el sujeto se desenvuelve, motivo por el cual se puede indicar que la competencia social es, en sí, una competencia ajustada al contexto cultural. Al respecto, García-Bacete y González-Álvarez (2010) señalan que la niñez aceptada por sus pares puede considerarse socialmente competente y la calidad de sus relaciones un índice de su competencia social.

2 Nelly Gromiria Lagos-San Martín y Ximena Soto-Soto

Los artículos de la Revista Electrónica Educare del Centro de Investigación y Docencia en Educación de la Universidad Nacional, Costa Rica, se comparten bajo términos de la Licencia Creative Commons: Reconocimiento, No Comercial, Sin Obra Derivada 3.0 Costa Rica. Las autorizaciones adicionales a las aquí delimitadas se pueden obtener en el correo: educare@una.cr 
Del Prette y del Prette (2008) sostienen que la competencia social es la capacidad para organizar pensamientos, sentimientos y acciones en función de objetivos y valores propios, dentro de un escenario situacional y cultural. Al ser la escuela el mejor escenario situacional para el desarrollo de relaciones interpersonales (Andrés, Barrios y Martín, 2005), la infancia es la época en que más conveniente resulta su estudio.

Las relaciones positivas con sus iguales favorece en la niñez una adaptación escolar satisfactoria y, por ende, un mejor ajuste psicoeducativo (García-Bacete, 2006; Gresham, 2011). Del mismo modo, las personas rechazadas o ignoradas por sus compañeros o compañeras o individuos controvertidos, en sus relaciones están más expuestos a sufrir desajustes psicosociales presentes o futuros (Inglés, Aparisi, García-Fernández, Núñez y Martínez, 2016; Pichardo, García, Justicia y Llanos, 2008).

La evaluación de las relaciones entre iguales es una temática que preocupa tanto a equipos investigadores como educadores. Ello, porque es en sí misma una temática relevante y, también, porque incide en el aprendizaje escolar. García-Bacete y González-Álvarez (2010) sostienen que los procedimientos de evaluación sociométrica entregan información fiable de los comportamientos sociales de los sujetos a través de las nominaciones entre pares; permite, además, la caracterización de los distintos niveles de complejidad de las relaciones diádicas de amistad o enemistad o a nivel de grupos o pandillas (García-Bacete, 2008).

Por tales motivos, este estudio se ha planteado como objetivo analizar las relaciones entre pares que generan los niños y niñas de la región de Ñuble y determinar, así, la presencia de distintas tipologías sociométricas diferenciadas por sexo y curso académico. Esto, porque se considera que analizar las competencias sociales del estudiantado en función del sexo y del curso académico es un buen punto de partida para generar intervenciones ajustadas a los requerimientos contextuales. Adicionalmente, se detectarán los índices de amistad y enemistad que se presentan en las aulas de esta región de Chile, para considerar estas tipologías dentro de un marco de referencia.

\section{Marco teórico o referencial}

Los primeros indicios sobre análisis sociométricos se encuentran en Moreno (1934). Esta herramienta conceptual y metodológica permite descubrir el funcionamiento de los grupos a partir de las fuerzas de aceptación y rechazo generados al interior de ellos. Muñoz, Moreno y Jiménez (2008) plantean un modelo triangular que explica este funcionamiento. En él, se valora la aceptación y el rechazo por separado, puesto que se considera la aceptación no como opuesta al rechazo, sino como contraria a la "no aceptación", y el rechazo no como contrario a la aceptación sino como "ausencia de rechazo", con lo cual aparece la indiferencia como una tercera dimensión ubicada en el centro de la aceptación y el rechazo. Desde este modelo, la 
doi: http://doi.org/10.15359/ree.24-1.8

URL: http://www.una.ac.cr/educare

CORREO: educare@una.cr

suma de nominaciones positivas recibidas por un sujeto dentro de un grupo corresponde a la aceptación que tiene este sujeto en el grupo y la suma de nominaciones negativas a una medida de rechazo; la ausencia de nominaciones positivas y negativas revelaría un índice de indiferencia, que da lugar a las personas olvidadas o ignoradas. A las categorías de preferido, rechazado e ignorado, se agregan la categorías de controvertido y promedio, categorías obtenidas por los sujetos que obtienen nominaciones tanto positivas como negativas. La diferencia entre estas últimas está en la cantidad de nominaciones de ambos tipos.

Enlaescuela, niños, niñasyadolescentes estableceny mantienen relaciones interpersonales para desarrollar su identidad social y su sentido de pertenencia (Jadue, 2001). El contacto cotidiano, estable y duradero, lleva a relaciones de amistad, entendiendo esta como un vínculo afectivo, construido voluntariamente entre las personas para favorecer el apoyo emocional entre los sujetos involucrados (Lanza, Moreno, de Diego, Ruiz y Moreno, 2012). Durante la niñez, se considera que la amistad es un vínculo necesario para el crecimiento personal, ya que sus beneficios se visualizan a nivel cognitivo, social y afectivo (Martín y Muñoz, 2009).

Las consecuencias de un determinado estatus sociométrico en la niñez suelen afectar de una manera muy marcada (Gifford-Smith y Brownell, 2003). Así, la conducta preferida en esta etapa es más amistosa y colaborativa; y la de niñez rechazada, más agresiva, oposicionista y demandante (Moreno, Estévez, Murgui y Musitu, 2009). Respecto de las niñas y los niños que se ignoran, se ha señalado que tienden a retrotraerse más al hacerse invisibles para sus pares y que los individuos controvertidos presentan problemas de adaptación al grupo, ya que son objeto de rechazo por una parte del grupo (García-Bacete, Sureda y Monjas, 2010).

\section{Relación entre competencia social y otras variables psicoeducativas}

El funcionamiento social eficaz de una persona es explicado por ciertas capacidades básicas para percibir, comprender, utilizar y manejar, de mejor forma, las emociones propias y ajenas, es decir, es una competencia fuertemente asociada a la inteligencia emocional (Morán y Olaz, 2014). Pichardo et al. (2008) agregan que una adecuada competencia social no solo se asocia a éxitos socioemocionales, sino que también se asocia con mejores resultados escolares. Al respecto, García-Bacete et al. (2010) declaran que las relaciones entre iguales, en tanto sean adecuadas, contribuyen al autoconocimiento, facilitan el manejo de los conflictos y contribuyen al desarrollo de la autonomía personal.

Del mismo modo, se señala que la niñez implicada en dinámicas de bullying tiene un estatus social más bajo que las personas no implicadas, y son los sujetos victimizados quienes presentan más dificultades en sus relaciones interpersonales (Cerezo y Alto, 2010; del Barrio, et al., 2008; Monks et al., 2009). En estos estudios se respalda, además, la importancia del grupo de iguales en la formación y mantenimiento de conductas agresivas (Cerezo y Ato, 2010; Salmivalli, 
2010). En este sentido, se puede plantear que la percepción del clima social y la violencia cotidiana también se encuentra relacionada con la ansiedad escolar (Astor, Benbenishty, Zeira y Vinokur, 2002; Martínez, 2004; Thomas, 2010).

Esto, debido a que un funcionamiento socialmente ineficaz tiende a generar desajustes psicológicos, los cuales suelen asociarse con algunas dificultades mayores. Así pues, el rechazo de iguales puede ser un indicador de dificultades académicas, de fracaso y absentismo escolar (Buhs, 2005; Inglés et al., 2009). La incompetencia social se relaciona, como es obvio, con una baja aceptación grupal, así como también con rechazo escolar, aislamiento social por parte de iguales, bajo rendimiento escolar, expulsiones, baja autoestima, locus de control externo, depresión e indefensión, así como también con diversos problemas de salud mental (Pichardo et al., 2008).

\section{Método}

\section{Participantes}

En esta investigación participaron 772 estudiantes de 26 cursos de entre 26 a 35 individuos por aula, pertenecientes a 13 colegios de 8 comunas de la región de Ñuble, en Chile. De ellos 420 niños y 352 niñas ( $54.4 \%$ y $45.6 \%$ respectivamente), 250 estudiantes cursan $4^{\circ}$ año, 274 cursan $5^{\circ}$ año y 248 cursan $6^{\circ}$ año de educación primaria.

La selección de los centros y de los cursos académicos fue realizada al azar, utilizando la estrategia del muestreo por conglomerados, de tal modo que la población de colegios urbanos de la región de Ñuble se representara en este estudio.

\section{Instrumento}

El instrumento utilizado es el cuestionario sociométrico de nominaciones entre iguales, el cual evalúa las competencias sociales entre pares, con base en 5 tipologías sociométricas: estudiantado rechazado, estudiantado ignorado, estudiantado controvertido, estudiantado preferido y estudiantado medio. Este instrumento aporta información adicional sobre las relaciones sociales de un curso, el clima social del aula, la existencia de subgrupos y el tipo sociométrico de cada componente del grupo.

Las preguntas que respondió el estudiantado fueron: ¿Quiénes son los tres compañeros o compañeras de curso que elegirías como mejores amigos o amigas? Escribe una lista por orden de más a menos y explica por qué les elegiste ¿Quiénes son los tres compañeros o compañeras de curso, que menos te gustan como amigos o amigas? Escribe una lista por orden de menos a más y explica por qué les elegiste. 
doi: http://doi.org/10.15359/ree.24-1.8

URL: http://www.una.ac.cr/educare

CORREO: educare@una.cr

\section{Procedimiento}

Seleccionados los centros educativos, se realizó una entrevista con la persona directora y profesorado de los establecimientos escolares para exponer los objetivos de la investigación y promover su colaboración. Posteriormente, se envió una carta informativa a los padres y madres en la que se les solicitó el consentimiento informado, por escrito, con lo que autorizaban la participación de sus hijos o hijas en el estudio. Contando con la ayuda de la dirección y del profesorado respectivo, se procedió a la administración del cuestionario en cada curso. Primero se les indicó que escribieran los datos de identificación, luego se leyeron las instrucciones en voz alta, destacando la importancia de no dejar ítems sin contestar.

\section{Análisis de datos}

Los datos fueron analizados a partir del software SOCIOMET (García-Bacete y GonzálezÁlvarez, 2010).

\section{Resultados}

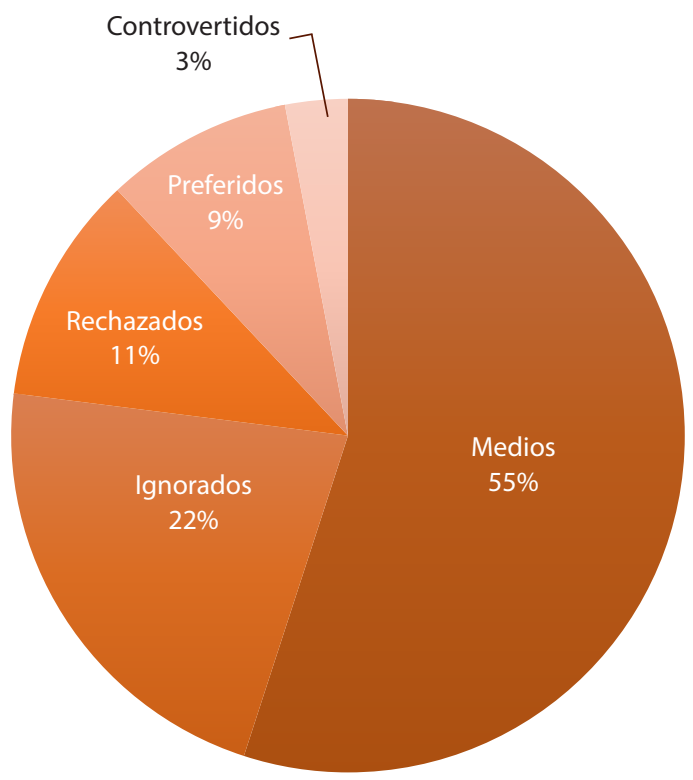

Figura 1: Tipología Sociométrica de la niñez evaluada. Nota: Elaboración propia. 
Los resultados muestran un mayor porcentaje de estudiantes clasificados en la tipología sociométrica estudiantado medio (más de la mitad de la muestra), hecho que resulta importante de destacar. Desde el plano negativo, también destaca el alto porcentaje de estudiantado ignorado, que sumado a estudiantado rechazado y estudiantado controvertido ocupan un 36 $\%$ de la muestra.

Estos datos, si bien estos no son los más deseables, cabe puntualizar que no se alejan de los reflejados en estudios previos, así como tampoco de lo que se esperaba encontrar (véase figura1).

Tabla 1: Tipologías sociométricas en función del sexo y curso académico del estudiantado

\begin{tabular}{ccccccccccc}
\hline & \multicolumn{2}{c}{ Rechazado } & \multicolumn{2}{c}{ Ignorado } & \multicolumn{2}{c}{ Controvertido } & \multicolumn{2}{c}{ Medio } & \multicolumn{2}{c}{ Preferido } \\
\cline { 2 - 10 } Curso & $\mathrm{H}$ & $\mathrm{M}$ & $\mathrm{H}$ & $\mathrm{M}$ & $\mathrm{H}$ & $\mathrm{M}$ & $\mathrm{H}$ & $\mathrm{M}$ & $\mathrm{H}$ & $\mathrm{M}$ \\
\hline $6^{\circ}$ & $4,85 \%$ & $4,84 \%$ & $14,92 \%$ & $6,85 \%$ & $2,42 \%$ & $0,40 \%$ & $27,82 \%$ & $29,44 \%$ & $5,65 \%$ & $2,82 \%$ \\
$5^{\circ}$ & $8 \%$ & $3,3 \%$ & $12,8 \%$ & $8,39 \%$ & $1,46 \%$ & $1,09 \%$ & $30,29 \%$ & $25,55 \%$ & $5,11 \%$ & $4,01 \%$ \\
$4^{\circ}$ & $6 \%$ & $5,2 \%$ & $13,2 \%$ & $10,4 \%$ & $2,28 \%$ & $2 \%$ & $23,2 \%$ & $28,8 \%$ & $5,2 \%$ & $3,2 \%$ \\
$\mathrm{~T}$ & $6 \%$ & $4,45 \%$ & $13,64 \%$ & $8,55 \%$ & $2,05 \%$ & $1 \%$ & $27,10 \%$ & $27,93 \%$ & $5,32 \%$ & $3,34 \%$ \\
\hline
\end{tabular}

Nota: $\mathrm{H}=$ Hombre, $\mathrm{M}=$ Mujer.

Nota: Elaboración propia.

Comparativamente, es posible observar un mayor porcentaje de niños rechazados, ignorados y preferidos, que de niñas en estas tipologías, hecho que se reitera también por curso académico. Por su parte, las niñas sobrepasan los porcentajes de los niños solo en la tipología "estudiantado medio", exceptuando en el $5^{\circ}$ año en que hay más niños que niñas en esta tipología (véase tabla 1).

Tabla 2: Cursos en los que no se presentaron tipologías sociométricas en el estudiantado

\begin{tabular}{ccccccccccc}
\hline & \multicolumn{2}{c}{ Rechazado } & \multicolumn{2}{c}{ Ignorado } & \multicolumn{2}{c}{ Controvertido } & \multicolumn{2}{c}{ Medio } & \multicolumn{2}{c}{ Preferido } \\
\cline { 2 - 11 } & H & M & H & M & H & M & H & M & H & M \\
\hline $4^{\circ}$ año & 1 & 1 & - & 1 & 2 & 3 & - & 1 & 2 & 2 \\
$5^{\circ}$ año & 2 & - & 1 & 1 & 7 & 6 & - & 1 & 2 & 2 \\
$6^{\circ}$ año & 2 & 2 & - & - & 7 & 3 & - & 2 & 4 & 4 \\
\hline
\end{tabular}

Nota: Elaboración propia. 
doi: http://doi.org/10.15359/ree.24-1.8

URL: http://www.una.ac.cr/educare

CORREO: educare@una.cr

Respecto del sexo y curso académico, resulta importante indicar una particularidad de algunos de los cursos evaluados, puesto que en cinco no se clasificaron niños rechazados (un curso de $4^{\circ}$ año, dos cursos de $5^{\circ}$ año básico y dos de $6^{\circ}$ año) y tres cursos en los que no se encontraron niñas rechazadas (un curso de $4^{\circ}$ año y dos cursos de $6^{\circ}$ año). Considerando la categoría estudiantado ignorado, en la mayor cantidad de cursos hay niños y niñas en esta, se exceptúan un curso de $5^{\circ}$ año, un curso de $4^{\circ}$ año y uno de $5^{\circ}$ año básico donde no hubo estudiantado ignorado. Respecto de la categoría controvertidas, se obseva una mayor cantidad de cursos en la que no se encuentran niñas clasificadas en esta tipología (dos cursos de $4^{\circ}$ año, siete de $5^{\circ}$ y siete de $6^{\circ}$ año), doce cursos en los que no se encontraron niños controvertidos (tres cursos de $4^{\circ}$ año, seis de $5^{\circ}$ y tres de $6^{\circ}$ año) (véase Tabla 2 ).

En la Figura 2, es posible observar que los cursos evaluados (26 en total), presentan índices de amistad superiores a los índices de enemistad, dato derivado de una mayor cantidad de reciprocidades positivas que negativas. Las reciprocidades de amistad se ubican en un rango de 14 a 56 coincidencias, las que, traducidas en índices, se ubican entre 0.7 y 1.60 . Las reciprocidades de enemistad, se ubican entre 2 y 22, lo que, reflejado en índices, equivale a un rango entre 0.08 y 0.71 .

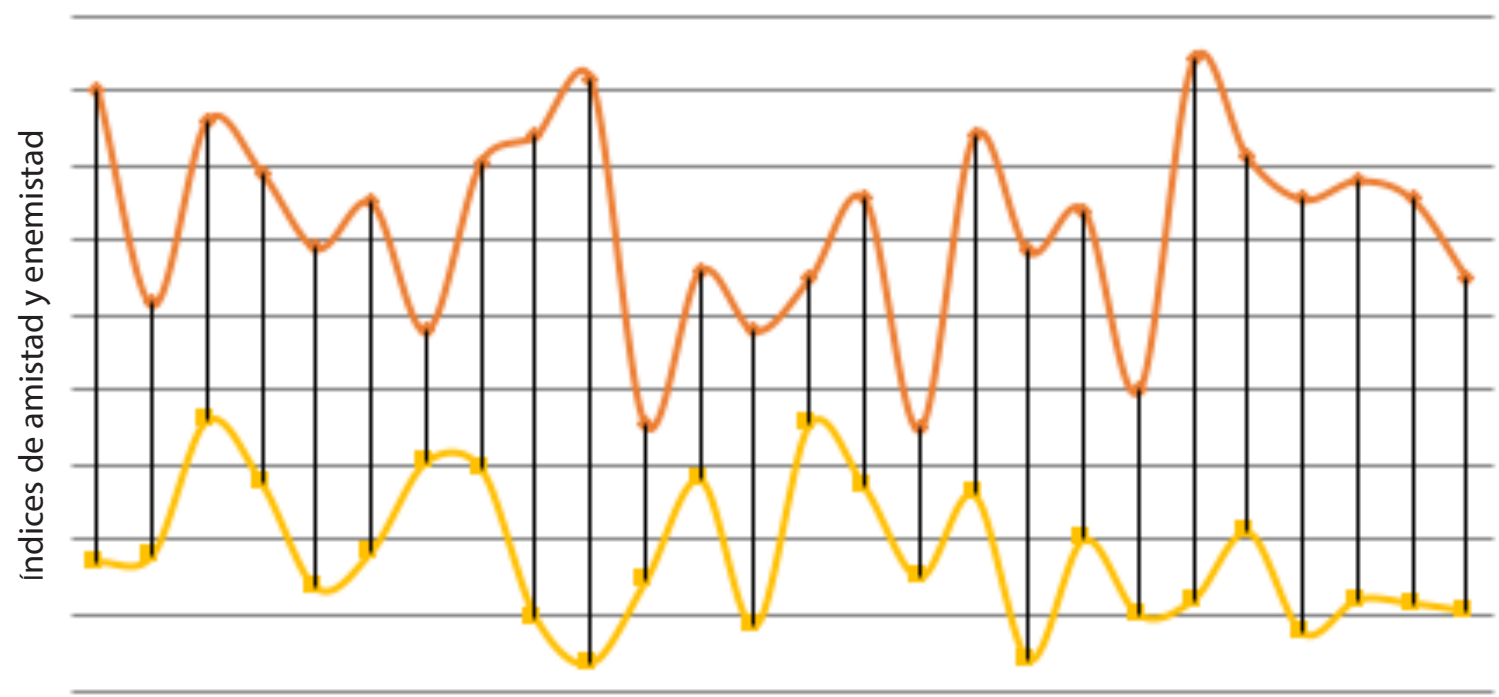

Cursos evaluados

Figura 2: Índices de amistad y enemistad presentados en los cursos evaluados.

Nota: Elaboración propia. 


\section{Discusión}

En su estudio, García-Bacete, Sureda y Monjas (2008) ofrecen una identificación de las tipologías sociométricas de niños y niñas con edades similares; señalan que la mayor parte se ubica en la categoría de "estudiantado medio", situación que se observó de manera similar, aunque en menor porcentaje (55.03 \%, versus $62.1 \%$ en el estudio mencionado). Del mismo modo, y coincidente también con este estudio, el menor porcentaje se encuentra ubicado en la tipología de estudiantado controvertido. Al respecto, García-Bacete (2006) informa un 4.2 $\%$ y en este estudio se obtuvo un $3 \%$. Finalmente, el orden de la distribución en las demás categorías es también semejante, solo se puede señalar como especificidad que en el grupo de este estudio se obtuvo un porcentaje muy superior de estudiantado ignorado ( $22 \%$ ) que en el estudio citado (12.4\%).

Lamentablemente, son escasos los trabajos que se encuentran en esta línea, sobre todo en Chile, por lo que los resultados de este no tienen muchos puntos de comparación que feciliten un análisis más completo.

En términos generales, García-Bacete et al. (2008) sostienen que niños y niñas son sociométricamente diferentes, mientras ellos son más rechazados y controvertidos, ellas son más ignoradas y medias. En este estudio, la situación se cumple en los niños; mientras que en las niñas solo parcialmente, puesto que las niñas superan en porcentaje a los niños en la tipología estudiantado medio, pero no en la categoría estudiantado ignorado.

\section{Conclusiones}

De acuerdo con la bibliografía, las consecuencias del estatus sociométrico están bien marcadas. En términos generales, la popularidad tiene efectos muy positivos para el sujeto y el rechazo efectos muy negativos (Gifford-Smith y Brownell, 2003). Los resultados obtenidos por García-Bacete (2008) informan que, con frecuencia, los sujetos rechazados se relacionan de manera agresiva, son demandantes de atención hacia sí mismos y no suelen reforzar positivamente a los demás (Monjas, 2007), también se ha señalado que muchas veces fanfarronean, mienten o bien comienzan a desarrollar sentimientos de inferioridad (Moreno et al., 2009). Desde este estudio, se puede señalar que las razones dadas para el rechazo de sus pares, siguen justamente estos patrones.

De lo señalado, es posible deducir la importancia que tiene la implementación de intervenciones que atiendan a la prevención de relaciones entre pares, basadas en el rechazo y la indiferencia, las que, además, deben ser ajustadas a los requerimientos detectados. En este sentido, los datos, según sexo, revelan que las relaciones entre los niños se caracterizan por ser controversiales, ya que entre ellos hay más preferidos, pero también hay más niños rechazados, ignorados y controvertidos que entre las niñas. 
doi: http://doi.org/10.15359/ree.24-1.8

URL: http://www.una.ac.cr/educare

CORREO: educare@una.cr

Según el curso académico, se puede señalar que los datos no son concluyentes; no obstante, se cree que favorecer una mejor relación entre pares a edades más más tempranas es siempre mejor. Aparte, es importante consignar, aquí, que el estudiantado rechazado por sus pares está distribuido en 18 de las 26 aulas, así como también el ignorado, lo está en 23 de estas 26 aulas. Se entiende, entonces, que en estos cursos es en donde se necesita de apoyo intencionado y constante, que les permita a estos sujetos desarrollar sus competencias sociales y así facilitar el desarrollo social en el entorno inmediato.

Desde un plano más general, se logra identificar más reciprocidad de amistad que de enemistad; no obstante, este elemento -que pudiera verse como positivo- no significa la inexistencia de reciprocidad negativa que, en sentido práctico, implica la presencia de climas de aula en los que se necesita favorecer el sentido de la amistad y el compañerismo.

En cuanto a ello, García-Bacete et al. (2013) advierten la necesidad de generar propuestas que favorezcan la integración social y escolar de estudiantes en situación de rechazo. Para esto postulan acciones integradas al currículo, que cuenten con el compromiso de toda la comunidad educativa y con el foco en la gestión social del aula, y el fortalecimiento en la calidad de la amistad. Las evidencias en favor de esta forma de trabajo indican que el desarrollo de una sana convivencia y la integración social de todo el alumnado se ha transformado en una herramienta eficaz para contrarrestar las situaciones de rechazo, exclusión y violencia experimentadas por gran cantidad de niños, niñas y adolescentes en las escuelas de hoy. Del mismo modo, constituyen una vía para el desarrollo de la inteligencia emocional y el aprendizaje escolar de todo el alumnado (Ortega, 2006).

\section{Agradecimientos}

Este estudio ha sido financiado por el proyecto 11160040 de CONICYT/FONDECYT.

\section{Referencias}

Andrés, S., Barrios, Á. y Martín, E. (2005). Evaluación de un programa de ayuda entre iguales en educación secundaria por medio de grupos de discusión: La opinión de los alumnos participantes. En J. A. del Barrio e l. Fajardo (Comps.) Nuevos contextos psicológicosy sociales en educación. Buscando respuestas (pp. 17-32). Santander: Psicoex.

Astor, R. A., Benbenishty, R., Zeira, A. y Vinokur, A. (2002). School climate, observed risky behaviors, and victimization as predictors of high school students' fear and judgments of school violence as a problem. Health Education \& Behaviour, 29(6), 716-736. doi: https:// doi.org/10.1177/109019802237940 
Buhs, E. S. (2005). Peer rejection, negative peer treatment, and school adjustment: Self-concept and classroom engagement as mediating processes. Journal of School Psychology, 43(5), 407-424. doi: https://doi.org/10.1016/j.jsp.2005.09.001

Cerezo, F. y Ato, M. (2010). Social status, gender, classroom climate and bullying among adolescents pupils. Anales de Psicología, 26(1), 137-144. Recuperado de https://revistas. um.es/analesps/article/view/92131/88721

del Barrio, C., Martín, E., Montero, I., Gutiérrez, H., Barrios, Á. y De Dios, M. J. (2008). Bullying and social exclusion in spanish secondary schools: National trends from 1999 to 2006. International Journal of Clinical and Health Psychology, 8(3), 657-677. Recuperado de http:// www.redalyc.org/articulo.oa?id $=33712016003$

del Prette, A. y del Prette, Z. A. P. (2008). Psicologia das relações interpessoais. Vivências para o trabalho em grupo (6a ed.). Petrópolis: Vozes.

García-Bacete, F. J. (2006). La identificación de los alumnos rechazados. Comparación de métodos sociométricos de nominaciones bidimensionales. Infancia y Aprendizaje, 29(4), 437-451. doi: https://doi.org/10.1174/021037006778849585

García-Bacete, F. J. (2008). Identificación de subtipos sociométricos en niños y niñas de 6 a 11 años. Revista Mexicana de Psicología, 25(2), 209-222. Recuperado de http://www.redalyc. org/articulo.oa?id=243016308002

García-Bacete, F. J. y González-Álvarez, J. (2010). Evaluación de la competencia social entre iguales. La sociometría y otras medidas. Madrid: TEA Ediciones.

García-Bacete, F.J., Jiménez, I., Muñoz, M.V., Monjas, M. I., Sureda, I., Ferrà, P., ... Sanchiz, M. L. (2013). Aulas como contextos de aceptación y apoyo para integrar a los alumnos rechazados. Apuntes de Psicología, 31(2), 145-154. Recuperado de http://www.apuntesdepsicologia. es/index.php/revista/article/view/317/291

García-Bacete, F. J., Sureda, I. y Monjas, M. I. (2008). Distribución sociométrica en las aulas de chicos y chicas a lo largo de la escolaridad. Revista de Psicología Social, 23(1), 63-74. doi: https://doi.org/10.1174/021347408783399480

García-Bacete, F. J., Sureda, I. y Monjas, M. I. (2010). El rechazo entre iguales en la educación primaria: Una panorámica general. Anales de psicología, 26(1), 123-136. Recuperado de https://dialnet.unirioja.es/servlet/articulo?codigo $=3133199$

Gifford-Smith, M. E. y Brownell, C. A. (2003). Childhood peer relationships: Social acceptance, friendships, and peer networks. Journal of School Psychology, 41(4), 235-284. doi: https:// doi.org/10.1016/S0022-4405(03)00048-7 
doi: http://doi.org/10.15359/ree.24-1.8

URL: http://www.una.ac.cr/educare

CORREO: educare@una.cr

Gresham, F. M. (2011). Análise do comportamento aplicada às habilidades sociais. En Z. A. P. del Prette y Z. A. P. del Prette (Eds.), Psicologia das habilidades sociais:Diversidade teórica e suas implicações (2a ed., pp. 17-66). Petrópolis: Vozes.

Inglés, C. J., Aparisi, D., García-Fernández, J. M., Núñez, J. C. y Martínez, M. C. (2016). Relación entre tipos sociométricos y metas académicas en una muestra de estudiantes españoles de educación secundaria. Universitas Psycholica, 15(1), 233-244. doi: https://doi.org/10.11144/ Javeriana.upsy15-1.rsma

Inglés, C. J., Benavides, G., Redondo, J., García-Fernández, J. M., Ruiz-Esteban, C., Estévez, C. y Huescar, E. (2009). Conducta prosocial y rendimiento académico en estudiantes españoles de educación secundaria obligatoria. Anales de psicología, 25(1), 93-101. Recuperdo de http://www.redalyc.org/articulo.oa?id=16711594011

Jadue, G. (2001) Algunos efectos de la ansiedad en el rendimiento escolar. Estudios Pedagógigos, 27, 111-118. doi: https://doi.org/10.4067/S0718-07052001000100008

Lanza, D., Moreno, G., de Diego, A., Ruz, C. y Moreno, A. (2012). Concepciones acerca de la amistad: Un estudio exploratorio con niños españoles e inmigrantes afincados en la Comunidad de Madrid. Familia y educación en un mundo de cambio, 2(1), 293-302. Recuperado de www. redalyc.org/pdf/3498/349832339030.pdf

Martín, E. y Muñoz, M. C. (2009). Un análisis contextual de la preferencia y el rechazo entre iguales en la escuela. Psicothema, 21(3), 439-445. Recuperado de http://www.psicothema. com/psicothema.asp?id=3651

Martínez, J. B. (2004). El miedo escolar de los/las alumnos/as y sus diferentes respuestas. Investigación en la Escuela, 54, 27-38. Recuperado de https://idus.us.es/xmlui/ handle/11441/61017

Monjas, M. I. (Dir.). (2007). Cómo promover la convivencia: Programa de asertividad y habilidades sociales (PAHS). Educación infantil, primaria y secundaria). Madrid: CEPE.

Monks, C. P., Smith, P. K., Naylor, P., Barter, C., Ireland, J. L. y Coyne, I. (2009). Bullying in different contexts: Commonalities, differences and the role of theory. Aggression and violent behavior, 14(2), 146-156. doi: https://doi.org/10.1016/j.avb.2009.01.004

Morán, V. E. y Olaz, F. O. (2014). Instrumentos de evaluación de habilidades sociales en América Latina: Un análisis bibliométrico. Revista de Psicología, 23(1), 93-105. doi: https://doi. org/10.5354/0719-0581.2014.32877

Moreno, D., Estévez, E., Murgui, S. y Musitu, G. (2009). Relación entre el clima familiar y el clima escolar: El rol de la empatía, la actitud hacia la autoridad y la conducta violenta en la 
adolescencia. International Journal of Psychology and Psychological Therapy, 9(1), 123-136. Recuperado de https://www.ijpsy.com/volumen9/num1/

Moreno, J. L. (1934). Who shall survive? A new approach to the problema of human interrelations. Washington, D.C.: Nervous and Mental Disease Publishing. doi: https://doi. org/10.1037/10648-000

Muñoz, V., Moreno, M. y Jiménez, I. (2008). Las tipologías de estatus sociométrico durante la adolescencia: Contraste de distintas técnicas y fórmulas para su cálculo. Psicothema, 20(4), 665-671. Recuperado de https://www.redalyc.org/articulo.oa?id=72720424

Ortega, R. (2006). La convivencia: Un modelo de prevención de la violencia. En A. Moreno (ed.), La convivencia en las aulas: Problemas y soluciones (29-48). Madrid: Secretaría General Técnica Ministerio de Educación y Ciencia.

Pichardo, M. C., García, T., Justicia, F. y Llanos, C. (2008). Efectos de un programa de intervención para la mejora de la competencia social en niños de educación primaria en Bolivia. International Journal of Psychology and Psychological Therapy, 8(3), 441-452. Recuperado de http://www.redalyc.org/articulo.oa?id=56080315

Salmivalli, C. (2010). Bullying and the peer group: A review. Aggression and Violent Behavior, 15(2), 112-120. doi: https://doi.org/10.1016/j.avb.2009.08.007

Thomas, S. P. (2010). A novel approach to decreasing bullying. Issues in Mental Health Nursing, 31(9), 551. doi: https://doi.org/10.3109/01612840.2010.506353 